\title{
Chemische Untersuchungen über das spezifische pigmentbildende Ferment der Haut, die Dopaoxydase. \\ Von
}

Br. Bloch

(Aus der dermatologischen Klinik in Basel. Direktor: Prof. Dr. Brano Bloch.) (Der Redaktion zugegangen am 27. November 1916.)

\section{Vorbemerkung.}

Die Lehre von der Entstehung des Pigmentes - speziell des natürlichen Hautmelanins - bildet noch heute, trotz den außerordentlich zahlreichen Untersuchungen der letzten Jahrzehnte, eines der dunkelsten Kapitel der normalen und pathologischen Physiologie.

Nur in bezug auf eine morphologische Streitfrage, nämlich den Ort der Pigmentbildung, beginnen sich die Ansichten insoweit zu klären, als durch histologische und experimentelle Studien verschiedener Forscher (Jarisch, Wieting und Hamdi, Loeb, Winkler, Kreibich und besonders Meirowsky) wenigstens das sicher gestellt zu sein scheint, daß die Hauptmenge des Hautmelanins in der Epidermis selber, d. h. also in Zellen epithelialer Abkunft erzeugt wird.

Ganz im Argen liegt dagegen noch die chemische Seite des Pigmentproblems. Obschon sich seit den ersten Versuchen von Nencki und seinen Mitarbeitern eine große Zahl von Forschern (ich nenne nur: v. Fürth, Spiegler, v. Zumbusch, Piettre, Gessard, Neuberg, Jäger, Eppinger) um die Ergründung der Natur des Pigmentes bemüht haben, so fehlt uns heute trotzdem noch jegliche sichere Kenntnis über seine Herkunft, Zusammensetzung und Bedeutung sowie über den Chemismus seiner Entstehung. Das geht schon aus dem Umstande hervor, daß die verschiedensten Körper von den einzelnen Forschern als Muttersubstanz des Melanins angesprochen werden: Tyrosin (Gessard, Durham), Adrenalin (Jäger, Neuberg, 
Untersuch. über das spez. pigmentbildende Ferment der Haut usw. 227

Meirowskyu.a.), Tryptophan(Spiegler, Eppinger, Fasal u.a.), daß ferner jeder Untersucher andere Analysenzahlen für das fertige Pigment findet als seine Vorgänger. Als Ursache der Pigmentbildung in der Haut ist vielfach, in Anlehnung an ähnliche Vorgänge bei Pflanzen und niederen Tieren, die Anwesenheit einer "Tyrosinase angenommen worden; doch steht diese Hypothese bis heute auf einem durchaus unsicheren Boden, denn es ist noch niemandem gelungen, ein solches Enzym (dem wie der gut fundierten Tyrosinase bei Pflanzen und niederen Tieren als Funktion die Bildung eines dunklen Farbstoffes aus Tyrosin zukäme) oder überhaupt ein Oxydationsferment in der Epidermis der höheren Tiere, also dort, wo das Melanin tatsächlich entsteht, nachzuweisen.1)

Durch meine eigenen Untersuchungen ist nun diese Sachlage eine völlig andere geworden und ich glaube behaupten zu dürfen, daß dadurch das Problem der Pigmentbildung in der Oberhaut des Menschen und der höheren Tiere in ein neues und definitives Stadium gerückt ist. Die wichtigsten Ergebnisse dieser Studien (bezüglich deren ausführliche Darstellung ich auf die Literaturangaben verweise) sollen hier, soweil sie zum Verständnis der vorliegenden Arbeit notwendig sind, kurz skizziert werden:

Werden überlebende Gefrierschnitte der Haut von Menschen oder Tieren mit einer $1-2 \%$ wässerigen Lösung von 3,4Dioxyphenylalanin ${ }^{2}$ ) ( $($ Dopa») behandelt, so tritt an bestimmten Stellen eine vitale Reaktion, die «Dopareaktion», auf.

1) Direkt positive Angaben über das Vorkommen einer Tyrosinase in der Haut von Säugern finden sich nur bei Durham; sie sind durch Untersuchungen von Ducrey aus meiner Klinik als irrig erwiesen.

2) Es kann entweder das natürlich vorknmmende, optisch aktive (linksdrehende) Dioxyphenylalanin verwandt werden, welches von Guggenheim zuerst aus Vicia faba rein dargestellt und in seiner Konstitution. aufgeklärt worden ist - die Substanz ist mir für meine Untersuchungen in höchst verdankenswerter Weise von der chemischen Fabrik F. HoffmannLa Roche \& Co. in Grenzach zur Verfügung gestellt worden - oder das synthetische, racemische Dioxyphenylalanin, das ich mir nach dem Verfahren von Fromherz und Hermanns selbst hergestellt habe. Über Unterschiede in der Wirkung dieser beiden Modifikationen vergl. vorliegende Arbeit. 
Die Dopareaktion besteht darin, daß das Dioxyphenylalanin durch Oxydation (t Kondensation) in einen dunkeln (rauchgrauen, dunkelbraunen oder tiefschwarzen) Körper, das «Dopamelanin», verwandelt wird; die Stellen, an welchen diese Reaktion stattfindet und das Dopamelanin gebildet und abgelagert wird, lassen sich daher, ebenso wie die Stärke der Reaktion, aus dem Vorhandensein und dem Grad der Dunkelfärbung genau unter dem Mikroskop verfolgen.

Die Oxydation des Dopa wird in den Granula der Leukocyten (in leukocytären Zellinfiltraten der Haut und in Blutausstrichen) sowie in den Schweißdrüsenzellen (und im Schweiß selbst) bedingt durch die Anwesenheit und Aktion des von Spitzer-Röhmann, Winkler, Schultze, Kreibich, Loele, v. Gierke u. a. entdeckten und studierten, seit längerer Zeit unter dem Namen Phenolase oder Polyphenoloxydase (Battelli-Stern) bekannten, nicht spezifischen Oxydationsfermentes. In diesem Falle stellt die Anwendung des Dopa nur eine neue Methode zum Nachweis einer bereits bekannten, intracellulären 0xydase (eben der Phenolase) dar, die allerdings allen bisher für denselben $Z$ weck benützten Methoden (der Adrenalinmethode Kreibichs, der Indophenolblaumethode von Spitzer-Röhmann, Winkler und Schultze usw.) weit überlegen ist.

Die eigentliche «Dopareaktion» sensu strictiori hat mit dieser 0xydation durch die Leukocyten- und Drüsenzellengranula nichts zu tun. Sie ist ein durchaus spezifischer Oxydationsprozeß, bedingt durch ein bisher völlig unbekanntes, intracelluläres Oxydationsferment, die Dopaoxydase. Sie manifestiert sich nur in epithelialen Elementen der Haut und zwar ausschließlich im Protoplasma (nicht Kern) der Basalzellen (bei starker Reaktion auch der Stachelzellen) der Epidermis, des Follikeltrichters, der Zellen der äußeren Haarwurzelscheide und der Haarmatrix, insoweit diesen Zellen die Fähigkeit, Pigment zu bilden, innewohnt. Das Protoplasma dieser Zellen ist also der ausschließliche Sitz des neuen Fermentes, der Dopaoxydase.

Die Form der reagierenden Zellen entspricht entweder 
derjenigen der normalen Epidermis- usw. Zellen, oder aber es handelt sich - dies trifft besonders häufig, aber nicht stets bei stark reagierenden Zellen $\mathrm{zu}$ - um eigentümliche dendritenartig gebaute Zellen, mit langen, verzweigten Protoplasmaausläufern, welche ebenfalls die 0xydase und mithin das dunkle Reaktionsprodukt enthalten, um sogenannte «Melanoblasten . Das in den fermenthaltigen Zellen gebildete und abgelagerte Dopamelanin ist darin entweder in diffusgelöster oder in granulärer Form, oder in beiden Modifikationen zugleich vorhanden.

Die Stärke der Reaktion (und mithin des Fermentes) ist sowohl beim Tier, als auch beim Menschen schon unter physiologischen Verhältnissen außerordentlich variabel. Die Schwankungen hängen $a b$ von individuellen und Rasseneigentümlichkeiten, von der Hautreaktion usw. und sind durch alle möglichen, von außen oder innen kommenden Reize bedingt. Es kommen schon bei gesunden, unter normalen Verhältnissen lebenden Individuen alle Abstufungen von negativer Reaktion (keine Andeutung von Bildung des Dopamelanins) bis zu maximal gesteigerter (ganze Epidermis = tief dunkles, undurchsichtiges Band) vor. Die Reaktion bleibt stets absolut aus in Haut und Haaren albinotischer Tiere, resp. in den weißen Partien gefleckter Tiere.

Sie kann an ein und derselben Epidermis, in demselben Hautschnitt von Zelle zu Zelle wechseln. Gewöhnlich reagiert nur ein mehr oder minder großer Teil der Basalzellen positiv, andere weniger oder auch gar nicht. Nur bei sehr starker Reaktion reagieren sämtliche Zellen der Malpighischen (und dann auch oft noch die der Stachelzell-) Schicht gleichmäßig und kräftig positiv. Im allgemeinen besteht schon unter normalen Umständen ein strenger Parallelismus zwischen Stärke der Dopareaktion und Grad der natürlichen Dunkelfärbung (Pigmentation) der Haut (Beispiel: Negative Reaktion albinotischer Haut und Haare).

Die Variationsbreite der Reaktion ist noch größer und mannigfaltiger unter pathologischen Bedingungen, indem die ' Stärke der Reaktion durch alle möglichen Einflüsse physikalischer, chemischer, infektiöser usw. Natur geändert werden 
kann. Auch diese Änderungen verlaufen im allgemeinen streng parallel mit den Änderungen in dem Pigmentationszustand der Haut. Die Stärke der Reaktion (= Menge und Aktivität des Fermentes) wird vor allem erhöht durch alle Reize aktinischer Natur (Thorium-X, chemisch wirksame Lichtstrahlen usw.), welche erfahrungsgemäß auch die Pigmentation der Haut am meisten befördern, ferner durch manche entzündliche Reize. Die Reaktion ist hochgradig im pigmentierten Naevus, negativ in den depigmentierten Flecken der Vitiligo.

Aus allen diesen und einigen anderen (hier nicht angeführten) Tatsachen läßt sich der SchluB ziehen, daß die Dopareaktion der Indikator ist für die Fähigkeit einer Zelle, normales Haut- und Haarpigment (Melanin) zu bilden. Die Ursache der positiven Dopareaktion (die "Dopaoxydase») ist zugleich die Ursache der Pigmenterzeugung. Nur dort, wo, und in dem Maße als die Dopaoxydase vorhanden ist, liegt die Möglichkeit vor, Melanin zu bilden. Das Dopaferment ist das Pigmentbildungsferment.

Damit ist das lang umstrittene Problem, in welchen Zellen und Zellbestandteilen und durch welche Zellfunktion das normale und pathologische Melanin der Haut und der Haare gebildet wird, gelöst.

Die nachfolgenden Untersuchungen bezwecken, zwei spezielle Punkte dieses Gesamtpigmentproblems, die in den übrigen, diesen Gegenstand berührenden Abhandlungen nur kursorisch angeführt worden sind, näher zu beleuchten. Der erste betrifft die Frage nach der Spezifität des Fermentes in chemischer Hinsicht; der andere handelt von dem Wesen der Dopareaktion und der Dopaoxydase und ihrer Beeinflussung durch verschiedene chemische und physikalische Einwirkungen.

I.

Der erste Teil der vorliegenden Studie hat den Zweck, den Aktionsbereich der Dopaoxydase in chemischer Hinsicht d. h. ihre Spezifität zu bestimmen. In der Fermentlehre sind solche Untersuchungen bekanntlich von großer 
Untersuch. über das spez. pigmentbildende Ferment der Haut usw. 231

Bedeutung, weil sie uns - wenigstens bei den sogenannten spezifischen d. b. auf einen Körper resp. eine Körperklasse eingestellten Fermenten - gestatten, die physiologische Funktion eines Enzyms klarzulegen. Diese Aufgabe ist allereings, wie in der Einleitung ausgeführt, für die Dopaoxydase bereits gelöst. Trotzdem verliert eine solche Untersuchung nichts von ihrem Werte; ist sie doch, abgesehen von ihrem allgemein biologischen Interesse, vorläufig das einzige Mittel, um der Frage über die Natur und Abstammung des natürlichen Melanins näher zu kommen.

Die Technik der Untersuchung war durch die oben angedeutete und anderweits in extenso geschilderte Methode des Nachweises der Dopaoxydase gegeben: Gefrierschnitte durch menschliche (in einigen Fällen auch tierische) Haut werden während 24 Stunden bei Zimmertemperatur und bei $37^{\circ}$ in folgende Flüssigkeiten gelegt:

1. Phys. NaCl-Lösung.

2. $1 \%$ wässerige Lösung von Dioxyphenylalanin (opt. akt. und racem).

3. 1\%o wässerige Lösung der Substanz, deren Angreifbarkeit durch die in der Haut enthaltene Dopaoxydase geprüft werden soll, nachher entwässert, aufgehellt und eventuell nach einer Nachfärbung (nach Unna-Pappenheim) eingebettet.

Die mikroskopische Untersuchung der Schnitte ergibt uns dann bei

1. : Verteilung, Menge usw. des nativen Pigmentes,

2. : Verteilung und Stärke des pigmentbildenden Fermentes (Dopaoxydase), gemessen an der Menge und Ausdehnung des Reaktionsproduktes (Dopamelanins),

3. : die Entscheidung, ob die vorhandene Dopaoxydase die fragliche Substanz unter Bildung eines melaninarligen Produktes zu oxydieren vermag, wodurch dann zugleich entschieden ist, ob der betreffende Körper als Pigmentmuttersubstanz überhaupt in Frage kommt oder nicht.

\section{A. Aromatische Stoffe mit AusschluB von Brenzkatechinderivaten.}

\section{Tyrosin.}

Die Untersuchung des Tyrosins ist nach verschiedener Richtung hin interessant. Der Körper zeigt einen dem Dopa vollständig analogen Bau; die Seitenkette ist dieselbe, der Ring unterscheidet sich nur durch den Mangel des Hydroxyls in $m$-Stellung. Die Substanz stellt ferner tatsächlich das Substrat eines bei Pflanzen (Bertrand) und niederen Tieren 
(Gessard, Biedermann, v. Fürth, Weindl) verbreiteten Oxydationsfermentes, der Tyrosinase, dar, welche aus ihr durch Oxydation ein pigmentartiges Produkt zu erzeugen vermag. Das hat dann weiter dazu geführt, auch die Pigmentbildung in der Haut der höheren Tiere durch die Einwirkung einer Hauttyrosinase auf Tyrosin zu erklären ( 0 ppenheim, Durham, Bittorf, Fischer u. Leschcziner, Gessard u. a.).

Unsere eigenen Untersuchungen mit einer 1-2\% Tyrosinlösung fielen demgegenüber vollständig negativ aus. Die Tyrosinschnitte unterscheiden sich nach 24 Stunden nicht von den in $\mathrm{NaCl}$ gelegenen Kontrollschnitten, während in den «Dopaschnitten, das Protaplasma der pigmentbildenden Epithelzellen (Malpighische Schicht, z. T. auch stratum spinosum) eine oft sehr starke diffuse und granuläre Schwarzfärbung aufweist.

Dem pigmentbildenden Ferment kommt somit keinerlei oxydierende Wirkung auf das Tyrosin zu und es kann daher dieses nicht als Muttersubstanz des natürlichen Hautmelanins angesehen werden.

Nebenbei sei erwähnt, daß auch die Granula von Leukocyten, welche in der Cutis der untersuchten Haut vorhanden waren, mit Tyrosin nicht reagierten. Es wird also diese Substanz auch durch die (Schultze-Winklersche) Phenolase der Leukocytengranula nicht angegriffen.

2. Paraoxyphenyläthylamin.

Nach Neuberg reagieren mit dieser Substanz die (tyrosinasehaltigen) Extrakte von Tintenbeuteln der Sepia officinalis, sowie Extrakte aus metastatischen Nebennierentumoren in der Leber. Unsere Versuche mit dopaoxydasehaltiger Haut verliefen vollständig negativ.

3. Hydrochinon.

Epidermis und Cutis sind diffus rostbraun gefärbt, in den fermenthaltigen (mit Dopa positiv reagierenden) Basalzellen keine Reaktion.

4. Homogentisinsäure.

Diese Substanz wurde zur Untersuchung herangezogen, weil sie ein normales Produkt des intermediären Stoffwechsels 
Untersuch. über das spez. pigmentbildende Ferment der Haut usw. 233

darstellt und sowohl in vitro als in vivo (Ochronose) in dunkelgefärbte Körper verwandelt werden kann, daher als Ausgangssubstanz des Melanins wohl in Betracht zu ziehen ist. Das Ergebnis war negativ.

5. Pyrogallol.

Haut, besonders Hornschicht, braun. Keine Reaktion in den Basalzellen.

6. Tryptophan.

Dieser Körper ist in neuerer Zeit mehrfach als Pigmentmuttersubstanz angesprochen worden. Zuerst und noch andeutungsweise von Spiegler, mit großer Entschiedenheit dann von Eppinger und Fasal. Diese Ansicht stützt sich auf einen Befund von Eppinger, wonach im Harn eines an Melanurie (infolge Melanosarkomatose) leidenden Patienten ein (nicht genau definierter) Pyrrolabkömmling vorhanden war, dessen Menge nach Einnahme von Tryptophan (kolorimetrisch durch Vergleichung mit einer bekannten Indollösung bestimmt) zunabm. Daß sich aus einem solchen Befunde - in einem Falle einer melanotischen Geschwulst - keine irgendwie sicheren Schlüsse auf die Entstehung des normalen Hautpigmentes ziehen lassen, bedarf kaum der Hervorhebung, umsoweniger als nach Blumenthal diese Pyrrolreaktion (Blaufärbung bei Zusatz von Nitroprussidnatrium, Lauge und überschüssiger Essigsäure) auch bei nicht melanotischen Tumoren vorkommen, bei melanotischen hingegen fehlen kann, also gar nichts mit der Melaninbildung als solcher $\mathrm{zu}$ tun hat. Die Ansicht, die Fasal ausspricht, daß «die Muttersubstanz des Pigmentes das Tryptophan ist», ist daher an sich schon sehr hypothetisch; sie wird nun durch den Ausfall unserer eigenen mit (aus Casein dargestelltem) reinem Tryptophan unternommenen Versuchen an dopaoxydasehaltiger Haut, endgültig widerlegt, da in den Basalzellen nicht die geringste Reaktion zustande kam.

\section{B. Brenzkatechinderivate.}

Nachdem festgestellt worden war, daß keine der untersuchten aromatischen Substanzen mit ein, zwei oder drei $\mathrm{OH}-$ 
Gruppen am Benzolkern mit dem pigmentbildenden Ferment der Haut eine Reaktion einging, welche derjenigen des Dioxyphenylalanins $z u$ vergleichen wäre, selbst wenn die Seitenketle, wie beim Tyrosin und Tryptophan, mit derjenigen des Dopa identisch ist, ergab sich die Aufgabe, Körper, welche mit dem Dopa in bezug auf den Kern übereinstimmen, also Brenzkatechinderivate, auf diese Eigenschaft hin zu prüfen. Bei der synthetischen Herstellung einiger bis dahin unbekannter Substanzen, welche hierbei zur Untersuchung kamen, hatte ich mich der Ratschläge des Herrn Dr. M. Guggen h eim zu erfreuen.

1. Brenzkatechin.

Sämtliche Hautelemente, besonders die Zellen sind braun. Keine Reaktion in den Basalzellen. Es ist also die Anwesenheit einer Seitenkette erforderlich, damit das Brenzkatechin durch das Ferment angegriffen werde.

2. Protokatechualdehyd. Resultat $=1$.

3. Protokatechusäure $=2$. Reaktion ebenfalls negativ ·

4. Kaffeesäure.<smiles>O=C(O)/C=C\CCC(O)O</smiles>

dargestellt aus Kaffeextrakt nach Hlasiwetz und in $1-2 \%$ Lösung nach Neutralisation mit $\mathrm{Na}$ bic. angewandt. Die Substanz unterscheidet sich vom Dioxyphenylalanin nur in der Seitenkette, die zwar ebenfalls aus drei Gliedern besteht, aber zwischen erstem und zweitem Kohlenstoffatom doppelte Bindung aufweist, an letzterem die $\mathrm{NH}_{2}$-Gruppe nicht besitzt (Akrylsäure statt $\alpha$-Aminopropionsäure). Die Schnitte färben sich mit dieser Substanz intensiv braun (am stärksten die Hornschicht); eine Reaktion findet nicht statt.

5. Adrenalin.

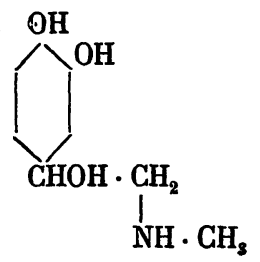


Die Prüfung des Adrenalins ist aus verschiedenen Gründen von besonderer Bedeutung. Einmal steht es, worauf zuerst Guggenheim aufmerksam gemacht hat, chemisch in naher Beziehung zum Dioxyphenylalanin, aus dem es - hypothetischer Weise - über die Stufe der Dioxyphenyl- $\alpha$-methylamino- $\beta$-oxypropionsäure

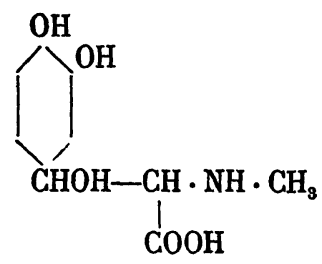

hervorgehen kann. Dann aber ist von einigen Forschern direkt die Ansicht ausgesprochen worden, daß das Adrenalin die Muttersubstanz des Hautpigmentes, sowohl unter physiologischen Verhältnissen als auch ganz besonders bei der infolge Nebenniereninsufficienz eintretenden Hyperpigmentation sei. Schon Neuberg konnte nachweisen, daß der Preßsaft einer melanotischen Lebergeschwulst (Metastase eines primären Nebennierentumors) Adrenalin unter Dunkelfärbung zu oxydieren vermochte; das Gleiche erzielte er mit einem Extrakt aus Tintenbeuteln. Jäger erhielt dasselbe Resultat mit melanotischen Tumoren von Schimmelpferden und schließt daraus ohne weiteres, daß auch für die Bildung des normalen Pigmentes in der Haut das Adrenalin die Muttersubstanz darstelle. Dieser Schluß ist aber, da Jäger Haut überhaupt nicht untersucht hat, keineswegs berechtigt. Nicht viel besser gestützt sind die sehr positiven Angaben von Meirowsky, Bittorf, Fischer und Leschcziner, wonach die Pigmentbildung in der Haut des Addison-Kranken auf die enzymatische Umwandlung des Adrenalins durch ein in vermehrter Menge vorhandenes Enzym (Tyrosinase, Oxydase) zurückzuführen sei (vgl. darüber Bloch und Löffler, Untersuchungen über die Bronzefärbung der Haut bei der Addisonschen Krankheit).

Allen diesen Hypothesen wird dadurch der Boden entzogen, daß das Adrenalin von der Dopaoxydase überhaupt nicht angegriffen wird und somit auch 
nicht als Pigmentvorstufe in Betracht kommen kann. Schnitte, welche die Dopaoxydase reichlich enthalten, färben sich zwar in Adrenalin braun, am stärksten das stratum corneum und die Haare; von irgend einer besonderen Reaktion in den Basalzellen kann aber nicht die Rede sein. Wir haben Gefrierschnitte von der Haut zahlreicher Menschen und Tiere daraufhin geprüft, immer mit demselben negativen Resultat.

Im Gegensatz hierzu vermag ein anderes tierisches Oxydationsferment, nämlich die Phenolase der Leukocytengranula, wie schon Kreibich gezeigt hat und ich bestätigen kann, das Adrenalin unter Bildung eines dunkelbraunen Farbstoffes zu oxydieren.

6. p-oxy-m-Methoxyphenylalanin.<smiles>CO[C@@H]1CCC[C@@H](NC(=O)O)C1</smiles>

Diese bisher nicht bekannte Substanz wurde folgendermaßen dargestellt:

Es wurde zunächst nach dem von Fromherz und Hermanns angegebenen Verfahren der synthetischen Darstellung des 3,4-Dioxyphenylalanins aus Vanillin (l) durch Kondensation mit Hippursäure die p-oxy-mMethoxybenz oylaminozimmtsäure (II) dargestellt und dieselbe durch Reduktion mit Natriumamalgam in das p-oxy-m-Methoxybenzoylphenylalanin (III) verwandelt. Aus dieser Verbindung wurde die Benzoylgruppe mit Salzsäure abgespalten, indem $10 \mathrm{~g}$ mit $60 \mathrm{ccm} \mathrm{HCl}$ von $1,12 \mathrm{spez}$. Gew. übergossen, das Ganze auf $100 \mathrm{ccm}$ Flüssigkeit gebracht und ca. 6 Std. im Rückflußkühler unter Kohlensäuredurchleitung über freier Flamme gekocht wurde. Nach dem Erkalten der Flüssigkeit scheidet sich die Benzoesäure ab und wird ausgeäthert. Der Rückstand wird im Vakuum zur Trockne eingedampft, mit Wasser aufgenommen, wieder verdampft, dann in wenig Wasser gelöst und mit Bicarbonat alkalisch gemacht. Beim Stehen der alkalischen Lösung (ca. 1 Tag) schieden sich schöne weiße Krystalle aus, die abgenutscht, gewaschen und getrocknet wurden. Aus der Mutterlauge lassen sich noch weitere Mengen des Körpers gewinnen, indem dieselbe im Vakuum zur Trockne eingedampft, der Verdampfungsrückstand mit Wasser aufgenommen, wieder zur Trockne eingedampft, mit wenig Wasser gelöst wird. Die wässerige Lösung wird neutralisiert, filtri ert, das Filtrat wieder mit Natr. bic. alkalisch gemacht, worauf sich beim Stehen wiederum dieselben Krystalle gewinnen lassen. Ausbeute ca $50 \%$ der berechneten. 
Die Reaktion wird iurch folgende Formeln reranschaulicht :<smiles>CO[C@@H]1CCC(O)C[C@H]1C=O</smiles>

I

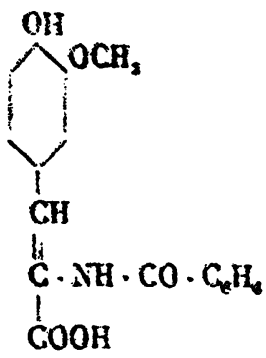

II

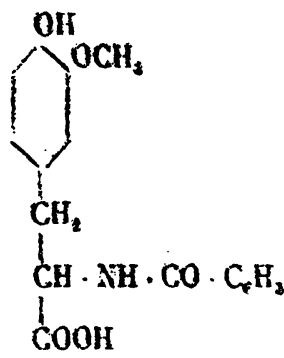

III<smiles>CO[C@H](O)CC1CCCC(C(=O)O)C1</smiles>

IV

Eine Stickstoffbestimmung der dargestellten Verbindung nach Slyke ergab:

$$
\begin{aligned}
& \text { Gelunden }=6.65 \% \mathrm{~N} . \\
& \text { Berechnet }=6.60 \mathrm{~N} .
\end{aligned}
$$

Die Mfethoxylbestimmung nach Zeissl ergab:

0,2190 g wasserfreie Subslanz gaben 0,$2 ; 60 \mathrm{~g} \mathrm{AgJ}$.

$$
\begin{aligned}
\mathrm{OCH}_{\mathrm{s}} . & \text { Gerunden }=11,63 \% \\
\text { Berechnet } & =11,410^{\circ} \mathrm{s} .
\end{aligned}
$$

Die Substanz bräunt sich bei $240^{\circ}$ und schmilzt bei $256^{\circ}$ (unkorr.) unter Zersetzung. Sie ist ziemlich leicht löslich in heißem Wasser; mit verdünntem Eisenchlorid gibt sie eine rasch rorübergehende hellbläuliche Färbung, die alsbald in hell-schmutzig-grün umschlägt, allmählich etwas dunkler wird und dann verblaßt. Zusatz von Soda zu dieser Mlischung gibt keine rote Färbung. Die typische Eisenchloridreaktion des Brenzkatechins und sciner Derivate ist also nicht melnr vorhanden. Bei der Spaltung des p-oxy-m-Nethoxybenzoylpbenylalanins mittels konz. $\mathrm{HCl}$ erfolgl also unter den obigen Versuchsbedingungen keine Abspaltung des Methyls der Methoxylgruppe - wofür übrigens auch der negative Ausfall der Dopareaktion einen Beweis bildet.

Mit einer $1 \%$ igen Lüsung dieses Körpers wurden nun eine ganze Anzahl Gefrierschuille der Haut verschiedener Menschen und Meerschweinchen in der üblichen Weise untersucht, welche sämtlich in den Basalzellen resp. in den Zellen der Haarmatrix mit Dopa einc stark positive Reaktion gaben, d. h. also reichlich Ferment besaßen. Die Epidermis fürble sich dabei leicht diffus grau. In den Basalzellen war vicht 
die geringste Andeutung irgend einer besonderen Reaktion zu konstatieren, dagegen ergaben die Leukocyten eines cutanen Infiltrates, das in einem der Präparate vorhanden war, eine zweifellose Reaktion, indem sich die Granula deutlich dunkelgraubraun färbten.

Es ergibt sich also aus diesen Versuchen, daB das Dioxyphenylalanin durch die Methylierung des in Metastellung befindlichen Phenolbydroxyls seinen Charakter gegenüber dem spezifischen Pigmentferment vollständig ändert, oder mit anderen Worten: dieses Ferment vermag ein Brenzkatechinderivat, auch wenn die Seitenkette durch eine $\alpha$-Aminopropionsäure dargestellt wird, nur dann zu oxydieren, wenn beide am Ring befindlichen $\mathrm{OH}-\mathrm{Gruppen}$ intakt sind.

Die Polyphenoloxydase der Leukocyten erweist sich auch hier als das viel weniger spezifische Ferment, indem sie fähig ist, diese Substanz noch zu oxydieren.

7. p-oxy-m-Methoxyphenylaminoessigsäure (I) und 3,4-Dioxyphenylaminoessigsäure (II)<smiles>COC1CCC(C(N)C(=O)O)CC1</smiles>

I<smiles>N[C@@H](C(=O)O)C1CCC(O)CC1</smiles>

II

Die Darstellung dieser, bis jetzt ebenfalls noch unbekannten Substanzen geschah anschließend an diejenige der Paraoxyphenylaminoessigsäure durch Fromherz folgendermaßen:

I. $17,5 \mathrm{~g}$ Vanillin werden in $400 \mathrm{ccm}$ Äther gelöst, $7 \mathrm{~g}$ Chlorammonium und $20 \mathrm{ccm}$ Wasser zugegeben, das Ganze in Eis gekühlt. Dazu wird dann, unter stetem Schütteln und Kühlen, allmählich eine Lösung von 7,5 g Cyankali in $30 \mathrm{~g}$ Wasser getropft und das Ganze schließlich genau wie bei Fromherz in einer Druckflasche auf der Schüttelmaschine einen halben Tag geschüttelt. Hierauf werden vorsichtig $120 \mathrm{ccm}$ Salzsäure und $70 \mathrm{ccm}$ Wasser zugesetzt, filtriert und das Filtrat im Vakuum 
zur Trockne verdampft. Der Rückstand wird in wenig Wasser gelöst, filtriert, mit einer konzentrierten Lösung von Natriumacetat versetzt. Aus der heißen Lösung krystallisieren feine, seidenglänzende, etwas grauweiße Nadeln. Eine weitere Portion läßt sich aus der Mutterlange durch Eindampfen im Vakuum noch gewinnen.

Der gewonnene Körper ist in kaltem Wasser sehr schwer, in heißem etwas leichter löslich. Mit Eisenchlorid gibt er eine rasch vorübergehende, schmutzig olivengrüne Färbung. Schmelzpunkt $240^{\circ}$ unter Zersetzung (unkorr.). Die Stickstoffbestimmung nach Slyke ergab:

$$
\begin{gathered}
0,0586 \text { Substanz enthalten } 4,21 \mathrm{mg} \mathrm{N} . \\
\text { Gefunden }=7,18 \% \mathrm{~N} . \\
\text { Berechnet }=7,07 \% . \mathrm{N} .
\end{gathered}
$$

.Die Methoxylbestimmung nach Zeissl ergab:

$$
\begin{aligned}
& 0,1414 \mathrm{~g} \text { Substanz ergeben } 0,1678 \mathrm{~g} \mathrm{AgJ} . \\
& \mathrm{OCH}_{3} . \text { Gefunden }=15,7 \% . \\
& \cdot \text { Berechnet }=15,6 \% .
\end{aligned}
$$

Es handelt sich hier also um p-oxy-m-Methoxyphenylaminoessigsäure. Die Ausbeute ist eine geringe.

II. Zur Darstellung der 3,4-Dioxypheṇylaminoessigsäure wurden 2,5 $\mathrm{g}$ der Substanz mit $10 \mathrm{ccm}$ Jodwasserstoffsäure $(1,70)$ im Kohlensäurestrom am Rückflußkühler auf dem Asbestteller erhitzt. Während des Kochens wurden noch $5 \mathrm{ccm}$ JH zugesetzt. Zur Entfernung der Jodwasserstoffsäure wurde im Vakuum im Kohlensäurestrom zur Trockne eingedampft, der Rückstand mit Wasser aufgenommen, unter Zusatz von etwas Blutkohle und Natrium bisulfuros. filtriert, das Filtrat mit Natriumacetat (ca. $5 \mathrm{ccm}$ gesättigte Lösung), darauf mit der gleichen Menge Alkohol versetzt. Es setzte sich über Nacht ein Niederschlag ab. Dieser wurde abgenutscht, mit Alkohol nachgewaschen. Der Niederschlag wog $1,6 \mathrm{~g}$, zeigte. eine dunkelgraue Färbung, wurde im Vakuum getrocknet, die färbende Substanz mit Äther entfernt, der Rückstand bestand aus einem schönen weißen krystallisierten Pulver und wog getrocknet $0,8 \mathrm{~g}$.

Diese Substanz gibt wie das 3,4-Dioxyphenylalanin typische Brenzkatechinreaktion. Mit verdünnter Eisenchloridlösung färbt sie sich dunkelgrün, auf Zusatz von Soda wird die grüne Lösung intensiv purpurrot.

$$
, 0,0375 \text { Substanz ergeben nach Slyke 0,00299 g N. }
$$

$$
\begin{aligned}
& \text { Gefunden }=7,97 \% \mathrm{~N} . \\
& \text { Berechnet }=7,65 \% \mathrm{~N} .
\end{aligned}
$$

Es liegt also 3,4-Dioxyphenylaminoessigsäure vor. 
Die Versuche mit p-oxy-m-Methoxyphenylaminoessigsäure ergaben, wie das nach den Resultaten unter $7 \mathrm{zu}$ erwarten ist, ein negatives Resultat: die Substanz wird durch das Dopaferment nicht verändert.

Dagegen war mir überraschend, daß auch die 3,4-Dioxyphenylaminoessigsäure mit dem Ferment nicht reagierte. Die Gefrierschnitte färbten sich in der Flüssigkeit leicht gelblich, am stärksten die Hornschicht. Weder in den Basalzellen noch in den Zellen der Haarmatrix konnte auch nur die geringste färberische Peaktion beobachtet werden; im Gegensatz dazu war das Protoplasma der Leukocyten rostbraun gefärbl, besonders bei $37^{\circ}$. Damit also ein Brenzkatechinderivat von dem Dopaferment oxydiert werden kann, ist nicht nur die Integrität der beiden OH-Gruppen erforderlich, sondern es ist notwendig, daß eine mindestens dreigliedrige Seitenkette vorhanden ist.

Die allgemeinen Schlüsse, die sich aus den vorhergehenden Versuchen in bezug auf das pigmentbildende Ferment in der Haut und in den Haaren ziehen lassen, sind folgende:

Die Dopaoxydase besitzt einen in chemischer Hinsicht außerordentlich spezifischen Charakter. Sie stellt von allen bisher bekannten intracellulären und in loco darstellbaren Oxydationsfermenten das weitaus spezifischste dar. Damit eine Substanz von diesem Ferment angegriffen werden kann, müssen nach den bisherigen Untersuchungen folgende Bedingungen erfüllt sein:

1. Die Substanz muß ein Brenzkatechinderivat sein.

2. Die beiden Phenolhydroxyle müssen intakt sein. Wird die in Metastellung zur Seitenkette befindliche OH-Gruppe z. B. durch $\mathrm{OCH}_{3}$ ersetzt, so bleibt die Reaktion aus, die Substanz ist für das Ferment unangreifbar geworden.

3. Die Seitenkette muß aus mindestens 3 Gliedern bestehen, also eine Propionsäure darstellen; ob auch höhere Homologe (Aminobuttersäure) eine Reaktion zulassen, ist unentschieden. Sie bleibt sicher aus, wenn die Aminopropionsäure durch eine Aminoessigsäure ersetzt wird. 
4. Zum Zustandekommen der Reaktion ist ferner die Anwesenheit einer Aminogruppe in der Seitenkette nötig. Fehlt dieselbe, wie bei der Kaffeesäure, wobei zwischen dem ersten und zweiten C-Atom doppelte Bindung vorhanden ist, so vermag das Ferment die Substanz ebenfalls nicht mehr zu oxydieren.

Es wird die Aufgabe weiterer Untersuchungen sein, festzustellen, ob sich die Spezifität des Fermentes noch enger und schärfer abgrenzen läßt. Es ist noch eine ganze Reihe von Variationen möglich, welche uns die Lösung dieser Aufgabe gestatten. Der Weg hierzu ist durch Untersuchungen klar vorgezeichnet. Es handelt sich darum, Substanzen darzustellen; welche von dem 3,4-Dioxyphenylalanin in dem einen oder dem anderen Punkte abweichen, und ihre Angreifbarkeit durch die Dopaoxydase nach der von uns befolgten Methodik zu studieren. Sehr interessant wird z. B. sein, das am Ring bromierte 3,4-Dioxyphenylalanin zu untersuchen; ferner den Ersatz der $\mathrm{NH}_{2}$-Gruppe in der Seitenkette durch eine OH-Gruppe; die $\beta$-Stellung der $\mathrm{NH}_{2}$-Gruppe statt der $\alpha$-Slellung, den Ersatz der Aminopropionsäure durch die nächst höheren Homologen usw.

Einstweilen bleibt der Satz zu Recht bestehen, daß von allen untersuchten aromatischen Körpern nur das 3,4-Dioxyphenylalanin durch das spezifische pigmentbildende Ferment (Dopaoxydase) der Haut und der Haare oxydiert werden kann und durch diese Oxydation in ein in allen indifferenten Lösungsmitteln unlösliches, dunkelbraunes bis schwarzes melaninartiges Produkt (Dopamelanin) umgewandelt wird.

Da wir zwei Modifikationen des Dioxyphenylanins kennen, die synthetische racemische und die natürlich vorkommende, linksdrehende, so ergab sich von selbst die Untersuchung über den Einfluß des stereoisomeren Aufbau des Dopamoleküls auf seine Angreifbarkeit durch die Dopaoxydase. Das Resultat dieser Prüfung, zu der sowohl menschliche als tierische Hautstücke verwendet 
wurden, ist noch kein abschließendes. Die meisten Hautpräparate, besonders die an sich stark reagierenden, also fermentreichen, zeigen keinen Unterschied, ob nun racemisches oder optisch aktives Dopa zur Reaktion benützt wird. Einige Schnitte dagegen, es handelt sich besonders um solche menschlichen Ursprungs mit nicht sehr intensiver Reaktion, gaben mit der linksdrehenden Modifikation deutlich einen stärkeren Ausschlag als mit der inaktiven. Es sind hier jedenfalls noch weitere Untersuchungen, womöglich auch mit der rechtsdrehenden Form erwünscht.

Eine weitere Frage, die ihrer Beantwortung noch harrt, ist die nach den Vorstufen des natürlichen Pigmentes. Die Annahme der früheren Autoren, nach welchen als solche Tyrosin, Adrenalin, Homogentisinsäure oder Tryptophan in Betracht kommen, sind durch unsere Versuche widerlegt; denn es ist unmöglich anzunehmen, daß eine Substanz als unmittelbare Pigmentvorstufe fungieren kann, welche durch das spezifisch pigmentbildende Ferment überhaupt nicht angegriffen wird. Da, wie wir gesehen haben, von allen untersuchten Körpern nur das 3,4-Dioxyphenylalanin dem Ferment Angriffspunkte darbietet und schon die geringsten Änderungen am Molekül die Eignung des Körpers zur Oxydation durch das Ferment zunichte machen, so liegt der Schluß sehr nahe, daß das 3,4-Dioxyphenylalanin selbst (oder doch eine ihm sehr nahestehende Substanz) mit der Vorstufe des natürlichen Hautmelanins identisch ist.

Diese Anschauung wird gestützt durch Resultate, welche Bloch und Löffler beim Studium der Bronzefärbung der Haut beim Addison-Kranken erhalten haben. Es hat sich nämlich gezeigt, daß die übermäßige Braunfärbung der Haut nach Ausfall der Nebennierenfunktion nicht, wie frühere Autoren (Bittorf u. a.) angenommen hatten, auf einer Vermehrung der Oxydase beruht, sondern daß sie hervorgerufen ist durch Überschwemmung des Organismus mit einer Vorstufe des Adrenalins, welche eben bei Zerstörung der Nebenniere nicht mehr, wie beim Normalen, durch die Funktion dieser Drüse in Adrenalin 
Untersuch. über das spez. pigmentbildende Ferment der Haut usw. 243

umgewandelt werden kann. Sie gelangt daher in vermehrter Menge in die Haut und wird dort durch die Dopaoxydase in Pigment übergeführt. Ein weiteres Moment, das zugunsten dieser Anschauung verwertet werden kann, sehen wir in dem zuerst von Bizzozzero geführten, später besonders von Schreiber und Schneider bestätigten Nachweis, daß überall da, wo Melanin vorhanden ist, und sogar dort, wo Vorstufen oder Abbauprodukte des Melanins zu vermuten sind, eine energische Reduktion des Silbernitrats zu konstatieren ist. Diese Reaktion läßt sich geradezu als Methode für die Darstellung pigmentierter Gebilde verwerten, da alle pigmenthaltigen Zellen durch das ausgefällte Silber dunkel gefärbt werden. Die Bilder decken sich in morphologischer Beziehung oft direkt mit den durch die Dopareaktion gewonnenen und es läßt sich hier so schön wie mit keiner anderen Methode demonstrieren, daß in derselben Zelle und in denselben Punkten, je nachdem man als Reagens das 3,4-Dioxyphenylalanin oder das Arg. nitric. (auch Goldsalze können an dessen Stelle treten) verwendet, ein oxydierender oder ein reduzierender Prozeß vor sich geht. Diese Erscheinung ist nur so zu erklären, daß die Dopaoxydase das Dioxyphenylalanin oxydiert, während das an derselben Stelle wie die Oxydase vorhandene Melanin resp. dessen Vorstufe die Metallsalze bes. $\mathrm{AgNO}_{3}$ reduziert. Im ersten Falle wird die Stelle sichtbar gemacht durch das schwarze Dopamelanin, im zweiten Falle durch die (viel gröber gekörnten, krystallinisch aussehenden) Ag-Partikelchen (daß es sich um solche handelt, läßt sich durch Behandlung mit Cyankali leicht nachweisen). Nun kommt dem Brenzkatechin und seinen Derivaten in besonderem Maße die Fähigkeit zu, Silbernitrat zu reduzieren; es spricht also auch diese Silberreduktion für die Annahme, daß das Pigment aus einem Brenzkatechinderivat entsteht.

Trotzdem möchte ich aber betonen, daß diese Annahme noch nicht strikte bewiesen ist; vorläufig kann sie nur als eine, allerdings durch die Tatsachen gut gestützte, Arbeitshypothese gelten. Eine Tatsache wird sie erst dann sein, wenn es gelingt, die Vorstufen des natürlichen Hautmelanins direkt darzustellen 
und $\mathrm{zu}$ identifizieren. Um zu diesem Ziel zu gelangen, sind verschiedene Wege möglich.

In einem vorläufigen Versuche haben wir, ausgehend von der Voraussetzung, da $B$ die Pigmentrorstufen im Serum von Addison-Kranken in vermehrter Menge auftreten, fermenthaltige Hautschnitte statt mit einer Dopalösung mit dem Serum eines solchen Kranken behandelt, um auf diese Weise das etwaige Vorhandensein von Dioxyphenylalanin in diesem Serum nachzuweisen. Das Resultat war ein negatives, was natürlich noch nichts gegen die Richtigkeit unserer Hypothese besagt.

Wenn sich unsere Voraussetzung, daB das 3,4-Dioxyphenylalanin, oder eine demselben sehr nahe stehende Substanz, mit der Pigmentvorstufe identisch ist, als richtig erweisen sollte, so wäre das natürlich nicht nur für das Problem der Pigmentbildung interessant, sondern es würde auch ein neues Licht werfen auf ein bis dahin sehr wenig bekanntes Kapitel des intermediären St offwechsels, indem damit die Körperklasse der Brenzkatechinderivate, von welcher uns bis jetzt im tierischen Organismus nur das Adrenalin bekannt ist (in Pflanzen spielt sie bekanntlich eine viel größere Rolle), eine neue Funktion zugewiesen bekäme. Das Pigment wäre dann gewissermaßen als eine Art Endprodukt des Brenzkatechinst off wechsels zu betrachten, indem, wie das der Addison lehrt, Brenzkatechinderivate nicht nur in der Nebenniere in Adrenalin umgewandelt, sondern auch durch die Tätigkeit der Dopaoxydase in der Haut in Pigment übergeführt werden können. Es wäre das ein um so interessanteres Kapitel des intermediären Stoffwechsels, als es hier zum erstenmal und durch eine neue Methode gelungen ist, nicht nur den genauen Ort der Umwandlung eines solchen Stoffwechselproduktes innerhalb der Zelle zu bestimmen, sondern sogar das Ferment, das diese Umwandlung bewirkt, direkt und in loco der Beobachtung zugänglich zu machen.

II.

Der zweite Teil der vorliegenden Untersuchungen beschäftigt sich mit den Eigenschaften des pigmentbildenden 
Untersuch. über das spez. pigmentbildende Ferment der Haut usw. 245

Fermentes, d. h. genau genommen mit seiner Resistenz gegenüber verschiedenen Eingriffen physikalischer und chemischer Natur.

Auch diese Untersuchungen sind wie die im ersten Teil geschilderten direkt in loco, d. h. an den das Ferment enthaltenden Hautschnitten vorgenommen worden, nicht, wie das sonst bei analogen Versuchen mit anderen Fermenten üblich ist, an fermenthaltigen Extrakten, resp. Lösungen. Einesteils ist es uns, wie aus der Arbeit von Ducrey hervorgeht, noch nicht gelungen, das Dopaferment einwandfrei aus dem Protoplasma der Basalzellen zu extrahieren (nur im Hautbrei kann es mit Sicherheit nachgewiesen werden); andernteils gibt die von uns befolgte Methode insofern zuverlässigere Resultate, als jede Schädigung des Fermentes durch die Extraktion dabei vermieden wird. Im einzelnen wurde folgendermaßen vorgegangen:

Fermenthaltige Hautschnitte wurden teils direkt während 24 Stunden mit Dopa behandelt, teils erst nachdem sie während längerer oder kürzerer Zeit (meist 24 Stunden) verschiedenen physikalischen oder chemischen Agentien ausgesetzt waren. Vor dem Einbringen der zweiten Serie in die Dopalösung wurde natürlich jeweilen jede Spur des zu prüfenden Agens durch Auswaschen usw. entfernt. Eine Vergleichung der bei den Serien unter dem Mikroskop ergibt dann - je nach dem stärkeren Ausfall der Dopareaktion, d. h. der Menge, und Verbreitung des durch die Dopaoxydase gebildeten dunkeln 0xydationsproduktes ( $₫$ Dopamelanins $»$ ) — ohne weiteres, ob die Wirkung des Fermentes durch die Vorbehandlung verstärkt, abgeschwächt oder aufgehoben worden ist. Wir schließen also aus der Menge des unter sonst gleichen Bedingungen durch die spezifische Fermenttätigkeit gebildeten Körpers auf die Stärke resp. Menge des vorhandenen und wirksamen Fermentes. Sehr kleine Ausschläge sind dabei, da es sich im Grunde um ein kolorimetrisches Verfahren handelt, natürlich nicht zu verwerten und im folgenden auch nicht berücksichtigt. Da es sich hierbei, wie ersichtlich, um Vergleichswerte handelt, so war die Untersuchung möglichst zahlreicher Schnitte derselben

Hoppe-Seyler's Zeitschrift f. physiol. Chemie. XCVIII. 
Provenienz erwünscht. Es wurde deshalb nicht nur die Haut menschlicher Individuen (mit durchweg starker Dopareaktion), sondern im großen Maßstabe auch Meerschweinchenhaut herangezogen, um den Einfluß der individuellen Variation, die gerade in bezug auf die Aktion des Dopafermentes, wie ich an anderer Stelle gezeigt habe, außerordentlich groB ist, auszuschalten. Da albinotische Tiere Dopaoxydase, wie schon erwähnt, nicht besitzen, und schwarzhaarige sich zu derartigen quantitativen Untersuchungen wegen der dunkeln Farbe und Massigkeit des natürlichen Pigmentes nicht eignen, wurden ausschließlich gelb resp. gelbbraun behaarte Nieerschweinchen verwendet. Bei diesen findet die Reaktion vor allem in der epithelialen Keimschicht des Haares (Haarmatrix) statt, die sich bei der Behandlung mit Dopa dunkelbraun bis braunschwarz färbt und dadurch außerordentlich deutlich von den nicht reagierenden oder nicht mit Dopa behandelten Haarbulbi absticht. Aus dem stärkeren, schwächeren oder negativen Ausfall dieser Reaktion ergibt sich die Intensität der Fermentwirkung. Daneben wurde jeweilen auch auf den Ausfall der Reaktion in der Keimschicht der Deckepidermis geachtet. In den von menschlicher Haut stammenden Präparaten wurde, wegen der Abwesenheit resp. Seltenheit der Haare, lediglich die Reaktion in den Basal- und Stachelzellen der Epidermis berücksichtigt. Es wurde sowohl optisch aktives als racemisches Dioxyphenylalanin verwendet und die Reaktion teils bei $37^{\circ}$ (hauptsächlich Menschenhaut) teils bei Zimmertemperatur (Meerschweinchenhaut) vorgenommen.

Da sich in einer großen Zahl der untersuchten Schnitte in der Cutis Leukocyten vorfinden, und die in den Leukocytengranulis vorhandene Schultze-Winklersche Phenolase, wie an anderem Orte gezeigt wird, ebenfalls mit Dioxyphenylalanin unter Bildung eines dunkeln Oxydationsproduktes reagiert, so konnte an Hand der vorliegenden Präparate zugleich die Beeinflußbarkeit dieses - von der Dopaoxydase wohl zu unterscheidenden, nicht spezifischen und in seiner Funktion noch durchaus unaufgeklärten Fermentes - verfolgt werden. Ich bemerke hier zusammen- 
fassend daß die meisten angewandten Reagentien auf beide Fermente in gleichem Sinne einwirken, daß sich aber in einigen Fällen (so bei Anwendung, von Erhitzung, Ammonsulfatlösung, sehr verdünntem Natr. bisulfurosum u. a.) deutliche Unterschiede in der Resistenz der beiden Fermente zeigen, die einen weiteren Beweis für ihre verschiedene Natur bilden.

Ich verzichte hier darauf, die Protokolle der einzelnen Versuche ausführlich wiederzugeben, und verweise auf die beigefügien Tabellen.

Die Resultate des zweiten Teils der vorliegenden Untersuchungen zusammenfassend, läßt sich sagen:

Das im Protoplasma der Keimzellen pigmentfähiger Haut und Haare vorhandene, die natürliche Pigmentbildung sowie die Dopareaktion verursachende Agens ist fermentartiger Natur: dafür spricht seine Thermolabilität, sowie seine geringe Resistenz gegenüber sogenannten Fermentgiften ( $\mathrm{HCN}, \mathrm{H}_{2} \mathrm{~S}$, Säuren und Alkalien, Phenylhydrazin usw.).

Dieses intracelluläre Enzym, die Dopaoxydase, dessen physiologische Funktion die Bildung des natürlichen Hautmelanins darstellt, ist ein Oxydationsferment, das seine Wirkung (Umwandlung des Dioxyphenylalanins in Dopamelanin) nur bei Anwesenheit von freiem Sauerstoff entfaltet. Wahrscheinlich ist mit dieser Oxydation, sowohl bei der Bildung des Dopamelanins wie bei derjenigen des natürlichen Pigmentes, zugleich eine Kondensation des Moleküls verbunden.

Die Dopaoxydase ist ein sehr labiles Ferment, das schon durch relativ sehr leichte Eingriffe $\left(\mathrm{H}_{2} \mathrm{O}\right.$, Toluol) geschädigt wird. Die Resistenz des Fermentes ist sowohl nach Tierart, als auch individuell verschieden; sie variiert auch etwas, je nachdem Basalzellen- oder Haarmatrixferment untersucht wird. Je stärker die primäre Dopareaktion, also Intensität resp. Menge des Fermentes, in einem Hautschnitte ist, desto resistenter erweist sie sich gegenüber schädigenden Einwirkungen, was sich nur mit der Annahme der stofflichen Natur des Fermentes vereinen läßt. 
Den einzelnen Einwirkungen gegenüber verhält sich die Dopaoxydase folgendermaßen:

1. Temperatur: Die Thermolabilität schwankt nach Tierart und auch individuell. Die Reaktion kann schon bei $57^{\circ}$ stark abgeschwächt oder aufgehoben sein. Maximal starke Reaktion in Menschenhaut wird selbst bei $80^{\circ}$ nicbt völlig vernichtet. Temperatur von $100^{\circ}$ zerstört die Fermentwirkung.

2. Austrocknung (bei langer Lagerung) vernichtet die Dopaoxydase nach vorheriger Abschwächung; ebenso wirkt Einbettung der Haut in Paraffin.

3. Röntgen- und ultraviolette Strahlen (Quarzlampe) schädigen leicht.

4. Destilliertes Wasser und physiologische $\mathrm{NaCl}-\mathrm{Lösung} \mathrm{schädigen} \mathrm{bei} \mathrm{längerer} \mathrm{Einwirkung.}$

5. Halb- und ganzgesättigte Ammonsulfatlösung vernichten das Ferment.

6. Äthylalkohol schädigt um so mehr, je geringer die Konzentration.

7. Fettlösende Mittel (Äther, Cloroform, Benzol usw.) haben keinen oder nur geringen Einfluß.

8. Säuren, Laugen (selbst in sehr geringer Konzentration) sowie proteolytische Fermente (Pepsin, Trypsin) vernichten das Ferment.

9. Oxydierende $\left(\mathrm{H}_{2} \mathrm{O}_{2}\right.$, Osmiumsäure $)$ sowie reduzierende $\left(\mathrm{H}_{2} \mathrm{~S}\right.$, Na bisulfuros., Phenylhydrazin; in geringem Grade Dextrose und Formaldehyd) Substanzen schädigen das Ferment stark resp. vernichten dasselbe.

10. Verschiedene Gifte: Blausäure vernichtet die D-O völlig. Fast ebenso nachteilig wirken Toluol und Diazobenzol. Cocain scheint schädigend $z u$ wirken, während Morphium und Chinin kaum oder nicht abschwächen.

\section{Literatur.}

Abderhalden und Guggenheim, Versuche über die Wirkung der Tyrosinase aus Russula delica. Diese Zeitschr., 1908. Bd. 54, S. 331.

Battelli und Stern, Die Oxydationsfermente. Ergebn. der Physiologie, 1912, Bd. 12, S. 96. 
Untersuch. über das spez. pigmentbildende Ferment der Haut usw. 249

Berdez und Nencki, Über die Farbstoffe melanotischer Sarkome. Arch. f. exp. Pharm. und Path., 1886, Bd. 20, S. 346.

Bertrand, Actions de la tyrosinase sur quelques corps voisins de la tyrosine. Compt. rend. de Soc. de Biol., 1907, Bd. 145, S. 1352.

Bittorf, Zur Frage der Pigmentbildg. bei d. Addison. Krankht. Arch. f. Pharmak. u. Ther., 1914, Bd. 75, S. 143.

Bizzozzero, Eine Methode zur Darstellg. v. Pigmenten. Münchn. med. Wschr., 1908, S. 2140.

Bloch, Br., Das Problem der Pigmentbildg. in der Haut. Arch. f. Dermat. u. Syph., 1917.

Bloch und Ryhiner, Histochemische Studien in überlebendem Gewebe 'über fermentative Oxydation und Pigmentbildung. Ztschr. f. d. ges. exp. Med., 1916.

Bloch und Löffler, Untersuchungen über die Bronzefärbung der Haut bei Morbus Addisoni. Dtsch. Arch. f. klin. Med.

Blumenthal, Bemerkungen zu einer Farbenreaktion in Harnproben bei Magencarcinom. Münchn. med. Wschr., 1916, Bd. 63, S. 530.

Chodat et Staub, Nouvelles recherches sur les ferments oxydants, 1907, Bd. 23, S. 265 und Bd. 24, S. 172.

Durham, One the presence of Tyrosinases in the skins of some pigmented Vertebrates. Proc. of the Royal Soc., 1904, Bd. 74, S. 310.

Ducrey, Über den Nachweis des pigmentbildenden Oxydationsfermentes in Hautbrei und Hautextrakten. I.-D. Basel 1916.

Eppinger, Über Melanurie. Biochem. Ztschr., 1910, Bd. 28, S. 181.

F a sal, Studien über Pigment. Biochem. Ztschr., 1913, Bd. 55, S. 393. - Derselbe, Über eine neue Darstellungsmethode des Pigmentes. Arch. f. Derm. u. Syph., 1914, Bd. 119, S. 317.

Fischer und Leschcziner, Diffuse Pigmentierung der Haut nach Schlußverletzg. i. d. Nebennierengegend. Derm. Wschr., 1915, Bd. 61, S. 1115.

Fromherz und Hermanns, Über den Abbau der aromatischen Aminosäuren im Tierkörper. Diese Zeitschr., 1914, Bd. 91, S. 194. Dieselben, Über das Verhalten der p-0xyphenylaminoessigsäure im Tierkörper. Ebenda, 1910, Bd. 70, S. 353.

v. Fürth, Physiolog. u. chem. Untersuchungen über melanotische Pigmente. Cbl. f. allgem. Path. u. path. Anat., 1904, Bd. 11, S. 618.

v. Fürth und Jerusalem, Zur Kenntnis der melanotischen Pigmente und der fermentativen Melaninbildung. Hofmeisters Beitr. 1907, Bd. 10, S. 131.

v. Fürth und Schneider, Über tierische Tyrosinasen etc. Ebenda 1902, Bd. 1, S. 229.

Gessard, Sur la formation du pigment mélanique. C. R. Acad. d. Sciences, 1903, Bd. 136, S. 1086. - Derselbe, Tyrosinase animale. C. R. Soc. Biol., 1902, Bd. 54, S. 1304. 
Guggenheim, Proteinogene Amine. Therapeut. Monatshefte 1913, S. 508. - Derselbe, Dioxyphenylalanin, eine neue Aminosäure aus Vicia faba. Diese Zeitschr., 1913, Bd. 88, S. 276.

Jäger, Die Entstehung des Melaninfarbstoffes. Virch. Arch., 1909, Bd. 198, S. 62.

Kreibich, Leakocytendarstellung im Gewebe durch Adrenalin. Wien. Klin. Wschr., 1910, S. 701. - Derselbe, Über das melanotische Pigment der Epidermis. Arch. f. Derm. u. Syph., 1914, Bd. 118, S. 837.

Loele, Oxydierende und reduzierende Zellsubstanzen. LubarschOstertag Ergebnisse, 1913, Bd. 16, S. 766.

Lutz, Zur Kenntnis der biologischen Wirkung der Strahlen auf die Haut, mit spezieller Berücksichtigung der Pigmentbildung. Arch. f. Derm. und Syph., 1917.

Meirowsky, Über den Ursprung des melanotischen Pigmentes. Leipzig 1908. - Derselbe, Über den Zusammenhang zwischen Hautorgan und Nebennieren. Münchn. med. Wschr., 1911, Bd. 58, S. 1005. - Derselbe, Zur Kenntnis der Fermente der Haut. Cbl. f. allgem. Path. u. path. Anat., 1909, Bd. 20, S. 301.

Nencki und Sieber, Weitere Beiträge zur Kenntnis der tierischen Melanine. Arch. f. exp. Pharm. u. Path., 1887, Bd. 24, S. 17.

Neuberg, Enzymatische Umwandlung von Adrenalin. Biochem. Ztschr., 1908, Bd. 8, S. 383. - Derselbe, Zur chemischen Kenntnis der Melanome. Virch. Arch., 1908, Bd. 192, S. 514.

Oppenheimer, Die Fermente, 4. Aufl. Leipzig 1913.

Piettre, Sur les pigments mélaniques de l'origine animale. C. $R$. Ac. d. Sc., 1911, Bd. 153, S. 782.

Röhmann und Spitzer, Über Oxydationswirkung tierischer Gewebe. Chem. Ber., 1895, Bd. 28, S. 587.

Schreiber und Schneider, Eine Methode zur Darstellung von Pigmenten usw. Münchn. med. Wschr., 1908, 1918.

Schultze, Die Oxydasereaktion an Gewebsschnitten. Zieglers Beitr., 1909, S. 127.

Spiegler, Über das Haarpigment. Hofmeisters Beiträge, 1904, Bd. 4, S. 40. - Derselbe, Über das Haarpigment nebst Versuchen über das Chorioidealpigment. Ebenda, 1907, Bd. 10, S. 253.

Weindl, Pigmententstehung auf Grund vorgebildeter Tyrosinasen.

Arch. f. Entwicklungsmechanik, 1907, Bd. 23, S. 632.

Wieting und Hamdi, Über die physiolog. u. patholog. Melaninpigmentierung. Zieglers Beitr., 1907, Bd. 42, S. 23.

Winkler, Der Nachweis von Oxydase in den Leukocyten. Folia Haematologica, 1907, Bd.4, S. 323. - Derselbe, Studien über Pigmentbildung. Arch. f. Entwicklgs. Mech., 1910, Bd. 29, S. 616.

v. Zumbusch. Beitr. z. Kenntnis des Sarkommelanins vom Menschen. Diese Zeitschr., 1902, Bd. 36, S. 511. 
Untersuch, über das spez. pigmentbildende Fermont der Haut usw. 251

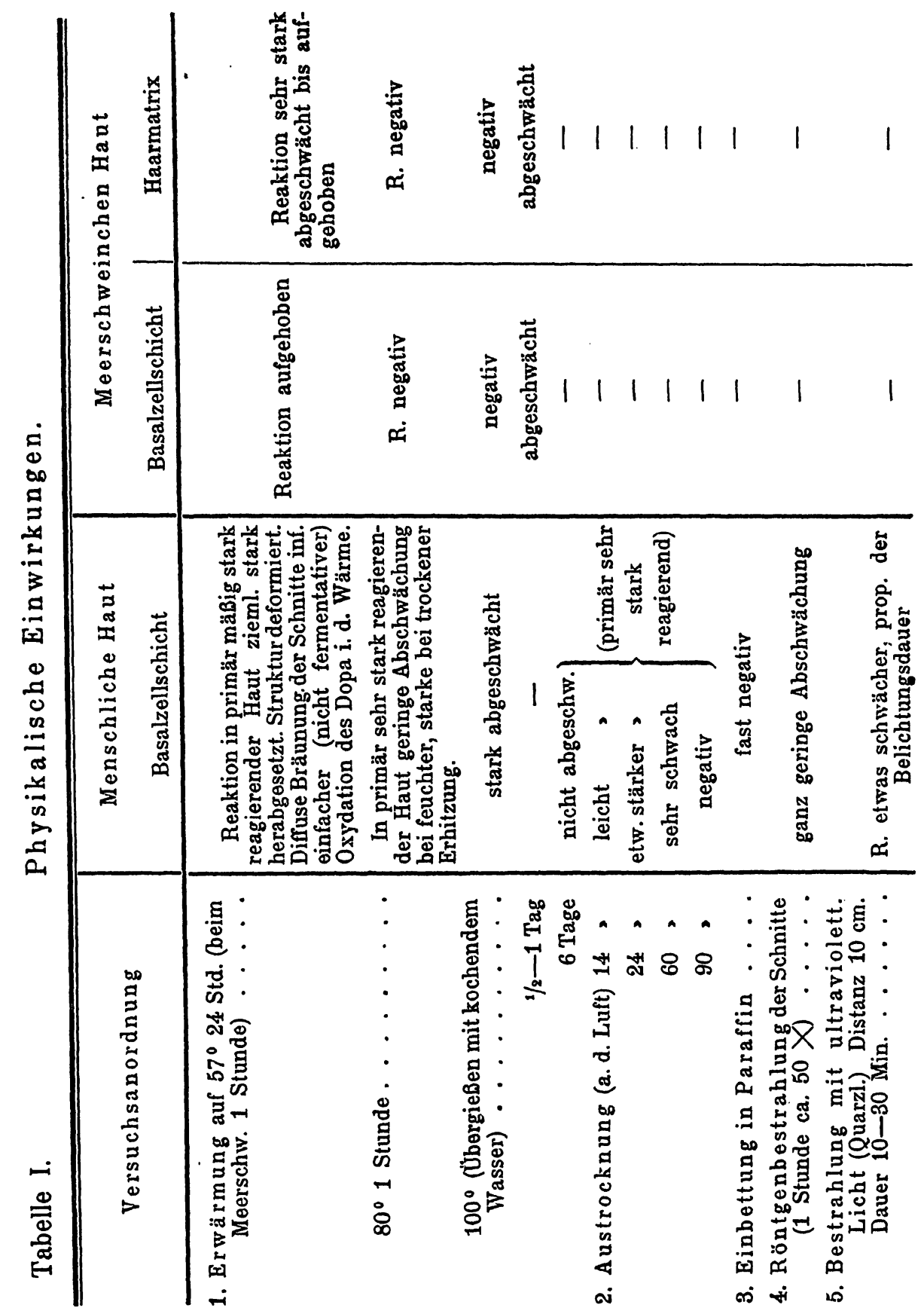


Br. Blocb,

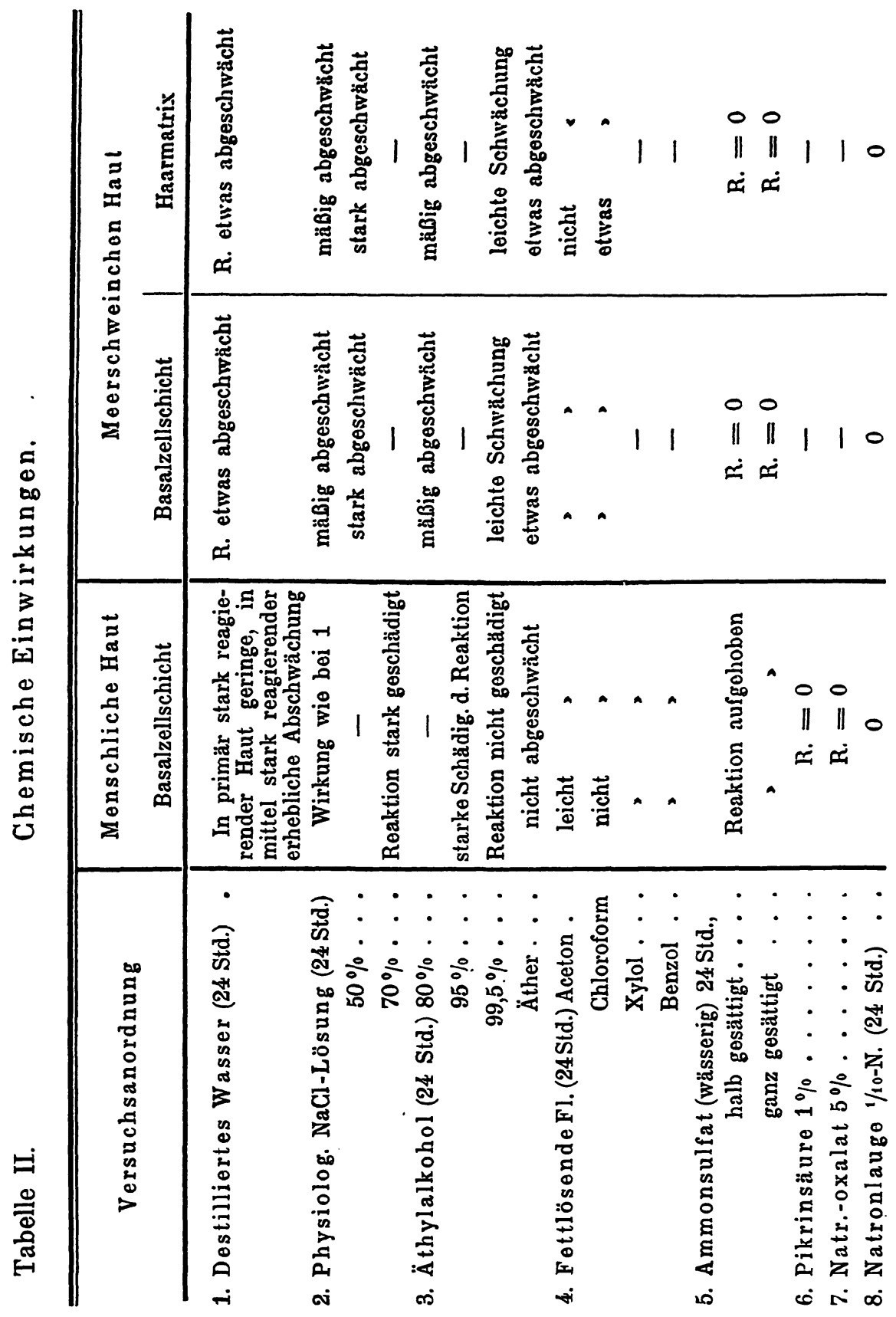


Untersuch. über das spez. pigmentbildende Forment der Haut usw. 253

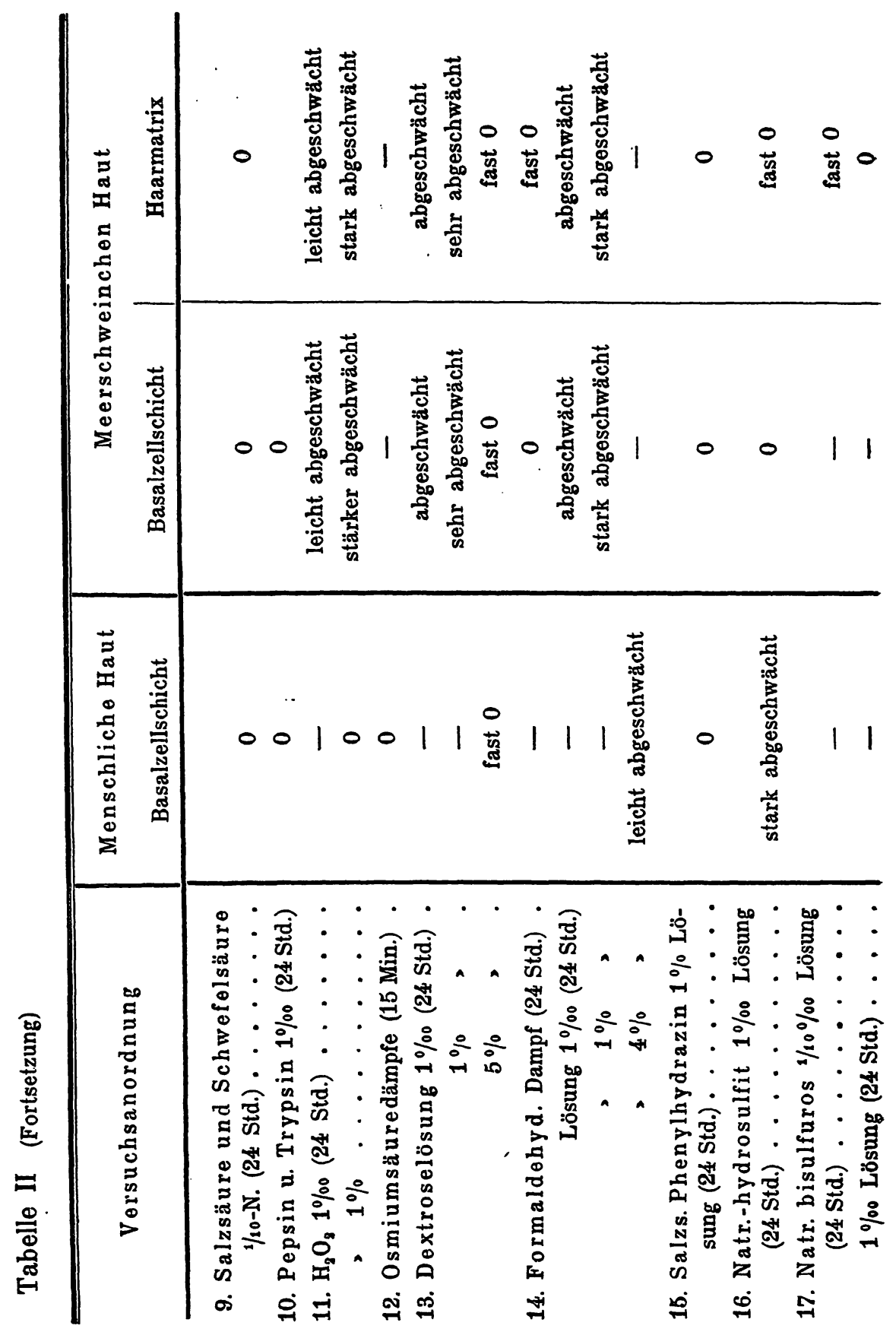


254 Br. Bloch, Untersuch. über das spez. pigmentbildende Ferment usw.

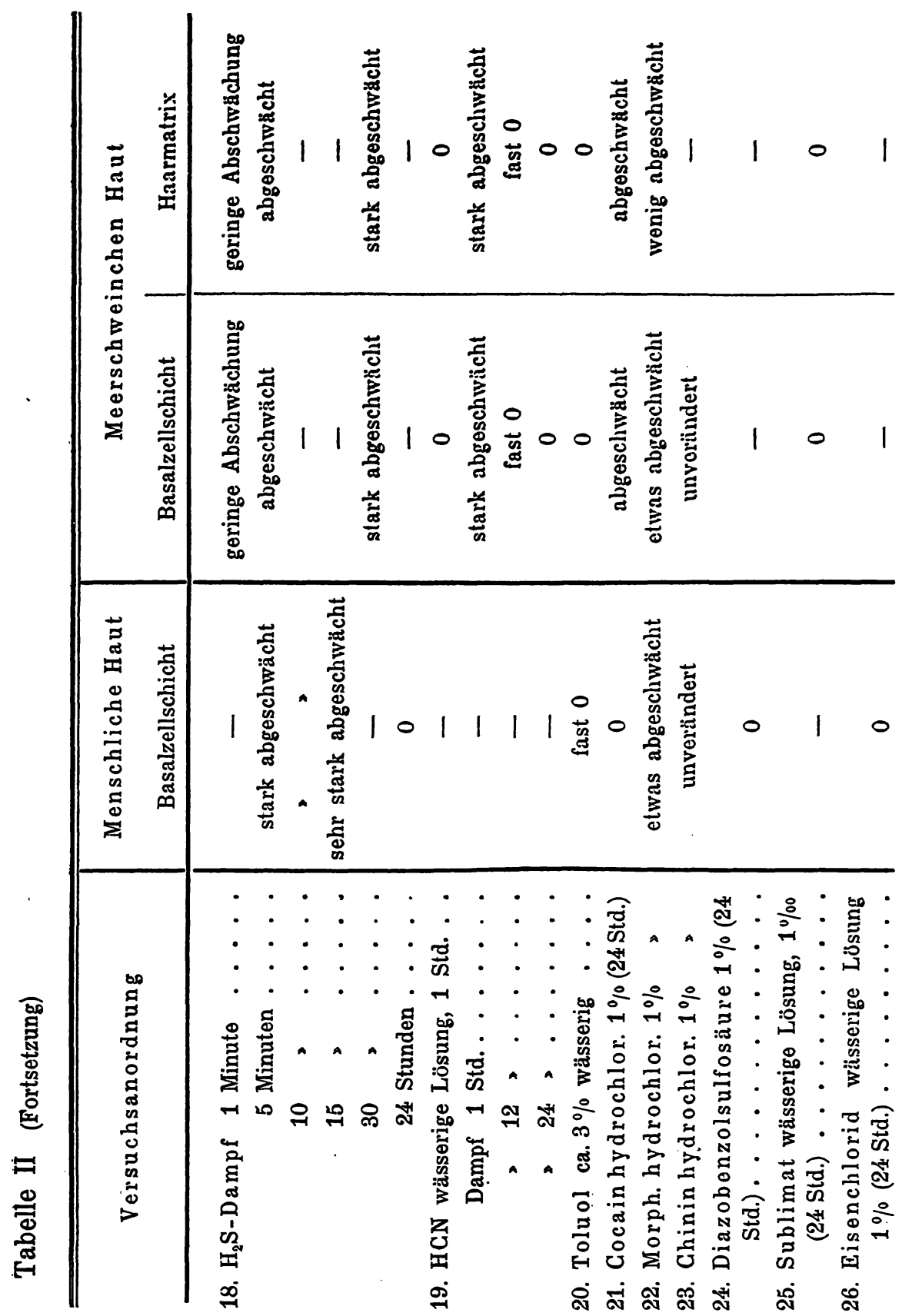

